\title{
FORUM
}

\section{The Cocked Hat}

\author{
from Charles H. Cotter
}

As every schoolboy learns, a point has position but no size, and a line has length but no breadth. Nevertheless, to a navigator concerned with fixing his ship, points and lines are represented on his chart as fine pencil marks.

The manner of representing the results of observations as pencil 'points' or 'lines', has undoubtedly led to the belief that a ship's position must be represented as lying at a point on a position line which is drawn on a chart. Nothing short of perfection in position lines is ever expected by most practical navigators, and two position lines crossing at a point are usually regarded by seamen as yielding a perfect position: and if three position lines meet at a point! . . there is seldom even the slightest doubt in the mariner's mind about the reliability of such a fix. On the other hand, three position lines which do not meet at a point are usually regarded as being something not quite respectable.

Now every seaman who has practised the art of navigation for more than a mere dogwatch, is well aware that all observations are prone to error, and many know that all position lines ought to be regarded with suspicion, but usually, the steps taken (if any) in dealing with any suspected unreliability in plotted position lines, are hesitant or uncertain. One may well wonder why this is so. The reason should be patent to any teacher in a British school of navigation which caters for the needs of merchant seamen. He will know that few navigators ever learn more about their craft than that which is necessary to pass M.O.T. examinations. Let it not be thought that this is a criticism of the examinations, for surely the task of examiners is to ensure that those to whom certificates are granted have at least a certain minimum knowledge sufficient to navigate a ship safely under normal circumstances.

The purpose of this article is to draw the attention of practical seamen to a small fragment of navigation theory, extremely simple to understand, but seldom if ever taught in navigation schools, for the sad and simple reason that the examiner does not include it in his syllabus of requirements. It is true that students preparing for the extra master examinations deal with at least a part of this work, but for obvious reasons this small and select group of students is excluded from the main body of practical navigators. By and large, British navigators of merchant ships are quite unaware of how possible errors in position lines should be treated methodically.

Errors in position lines obtained from astronomical observations are not generally serious, for these observations are usually made when the ship is in the open sea, far removed from the hazards associated with the shore. It is when practising the art of pilotage proper, or coastal navigation as it is called, that errors in position lines become really significant.

The most common method of fixing when in sight of land is by cross bearings. The compass bearings of three land- or sea-marks are observed; the compass 
error (if any) is applied in order to obtain the corresponding true bearings; and three position lines are laid down on the coastal chart of large scale. The three position lines occasionally meet at a point, and when they do, it is an occasion for mild rejoicing on the part of the observer. In most cases of fixing by cross bearings obtained from compass observations of three marks, the position lines do not meet at a point-they form a triangle of error known to seamen as a cocked hat.

The cocked hat in all cases reveals error. The error, whatever is its cause, results in one or more of the position lines being laid down in the wrong direction. It would be interesting to know just how the practical navigator handles the situation immediately following the appearance on his chart of a cocked hat. In this respect observers fall into one of four main categories.

A member of the first group in our simple classification would, on forming the cocked hat, curse the third position line and ignore it.

The second group includes those really practical seamen who realize that the lines they have drawn on their charts are, as the authors of the Institute's monograph Observational Errors put it, 'expressions of opinion and subject to all sorts of errors'. These practical navigators usually regard the ship's position as being at one corner of the cocked hat-the choice of corners being made according to circumstances.

The third group consists of men of long experience who have developed the seaman's sixth sense to a high degree, and regard every fix with the greatest suspicion. These navigators are wont to draw large and heavy-pencilled rings around their cocked hats, and having done so set their courses from a point on the ring to allow for maximum margin of error.

A fourth group includes the keen student of navigation-many of whom have read the excellent monograph by Anderson and Parker, and have given careful thought to the problem, and attempt to deal with it methodically and, therefore, scientifically.

The majority of navigators would fall into the second and third categories, those forming the first and fourth groups being small minorities - very small it is to be hoped in the case of the first group.

Now a man cannot be expected to handle a problem methodically unless the parts of the problem are understood perfectly. Let us therefore investigate methodically the three position lines which may form a cocked hat.

Any error which affects a position line, apart from stupid mistakes, or blunders as they are euphemistically called by statisticians, may be a combination of instrumental error (compass error or error in the adjustment of the azimuth mirror or circle); and observational error which cannot be foreseen (unsteady compass due to yawing, \&c.).

Instrumental errors are regarded as systematic errors, and if the same error affects all observations it can, if it is known, be offset.

If the compass error used to convert two compass bearings into true bearings is misapplied to the extent of $\epsilon^{\circ}$, it is a simple matter to show that the false position determined by crossing the two position lines obtained, will lie somewhere on the arc of a circle which passes through the observed marks and through the unknown true position.

In Fig. I, $T$ represents the true position and $X$ the false position determined by observing compass bearings of marks $A$ and $B$, and laying down the two position lines with an error of $\epsilon^{\circ}$. Clearly the line TX is a chord of the circle 
passing through $\mathrm{A}, \mathrm{B}$, and $\mathrm{T}$, for the angles at $\mathrm{A}$ and $\mathrm{B}$ standing on TX are equal.

Few navigators would rely on a fix obtained from two bearings only, as there would be no indication of the possibility of an error in the fix. It is customary to observe a third or check bearing, for if a cocked hat is formed, the observer will at least be aware that an error has occurred.

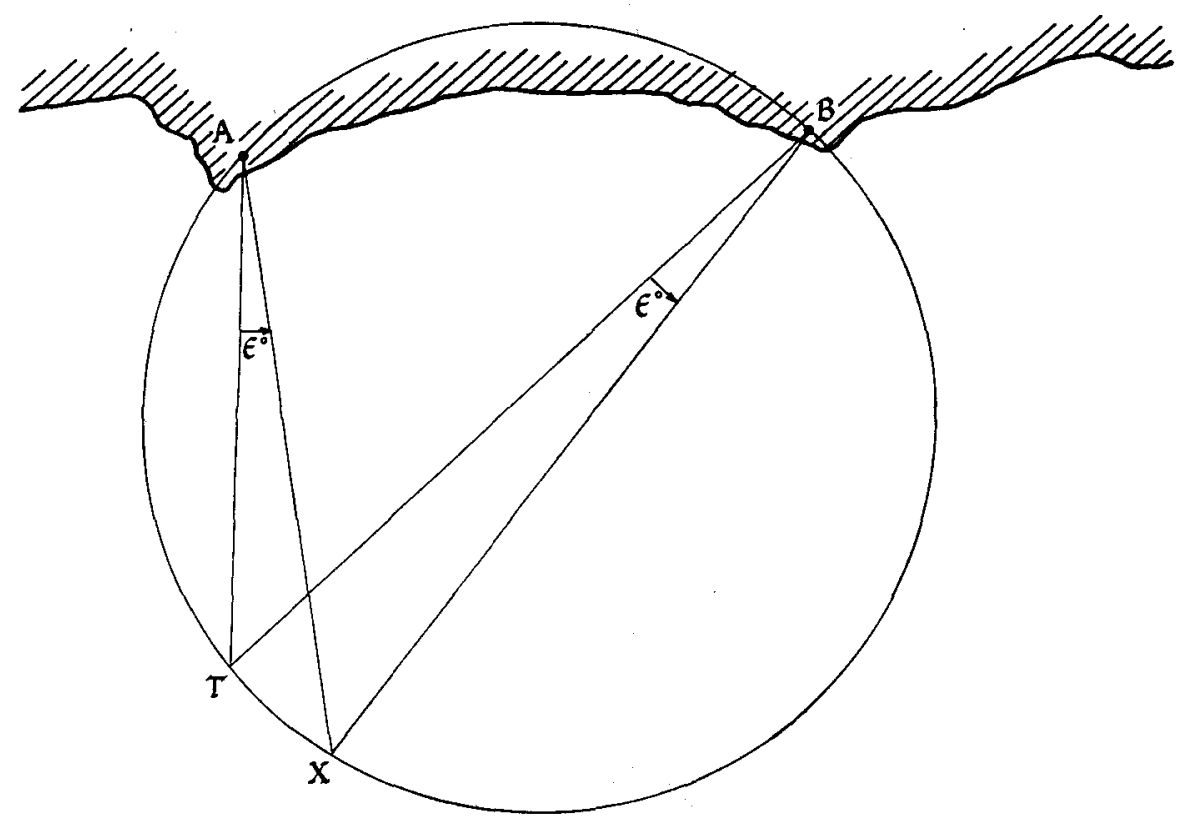

Fig. 1

A particularly insidious case occurs when the three position lines intersect at a point despite the fact that an unknown error exists. An error in any one (and only one) of three position lines will always result in a cocked hat; but an error in any two or in every one of the three, whether the errors are systematic or random, may result in the position lines meeting at a point. How natural (but how wrong) it is for the mariner to think that, if three position lines obtained from his own careful observations meet at a point, he has obtained a perfect fix. His attention has probably never been drawn to this possibility, and he has never realized that three straight position lines drawn from the charted positions of three observed marks can be made to meet at an infinite number of points, any one of which may be his 'perfect fix'. Fig. 2 should help to knock the idea that three position lines meeting at a point always produce a perfect fix, into a cocked hat.

In Fig. 2, T represents the true position of an observer, and $X_{1}$ is a false fix due to errors of $\epsilon_{1}, \epsilon_{2}$, and $\epsilon_{3}$ being applied to the three position lines as shown. $\mathrm{X}_{2}$ is a false fix due to errors of $\theta_{1}, \theta_{2}$, and $\theta_{3}$ being applied to the three position lines.

If the same error $\epsilon$ affects three position lines obtained from bearings of three marks, and the observer and the three marks are concyclic, an apparent perfect fix will result, for the position lines will clearly meet at a point.

The false fix $\mathrm{X}$ in Fig. 3 is due to an error $\epsilon$ affecting all three position lines. 
226

FORUM

VOL. XIV

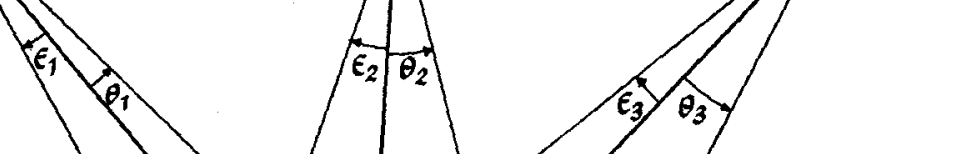

Fig. 2

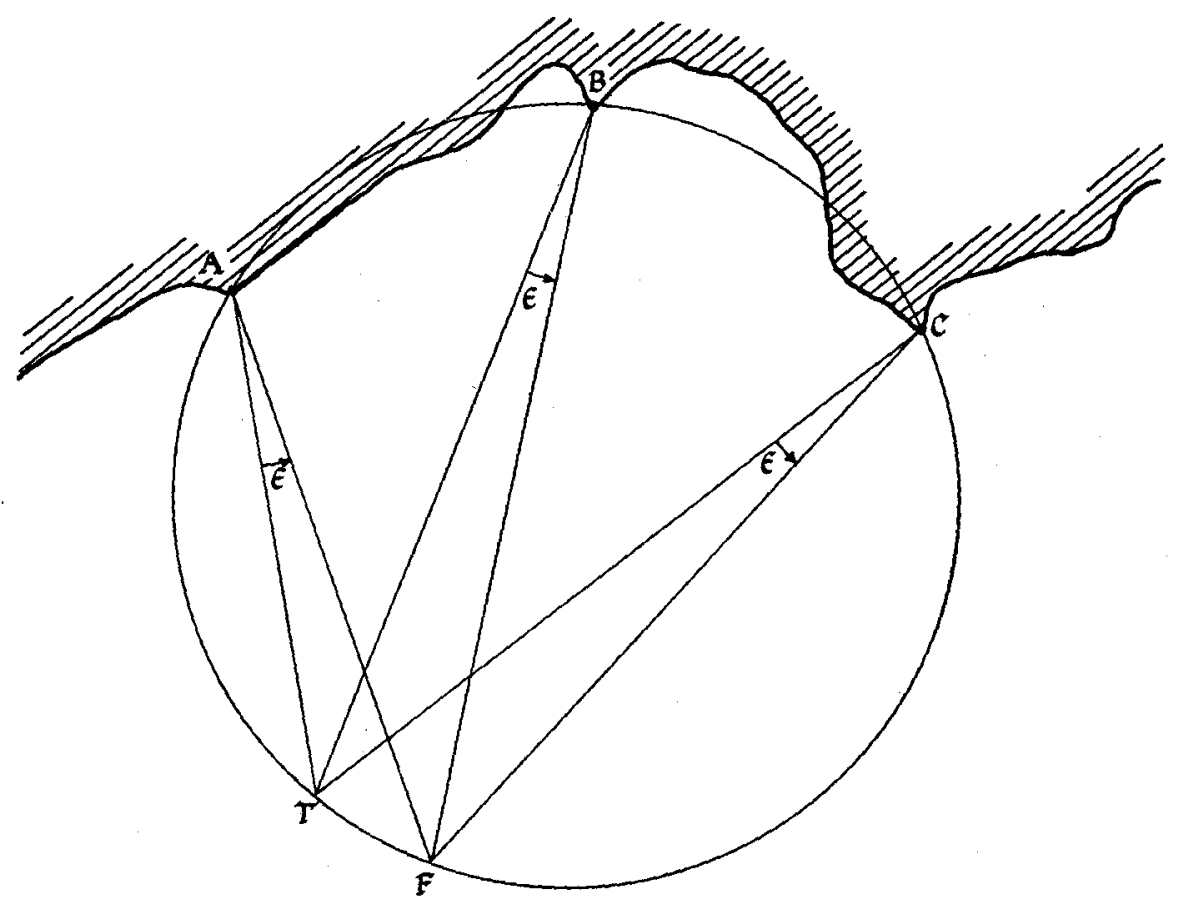

Fig. 3 
Because errors in laying down position lines are often regarded as being systematic errors common to all three position lines, the well-known and useful rule 'avoid choosing marks which are concyclic with the ship's position or nearly so', was undoubtedly formulated.

When fixing by cross bearings of three marks, an error in one position line always, and an error in two or three will sometimes, result in the formation of a cocked hat. Although when one is formed, most navigators regard the most likely position of the ship to be somewhere within a cocked hat, there is every likelihood that the ship's position is in fact, outside the cocked hat. The authors of the Admiralty Navigation Manual draw attention to this by stating that the chance of falling inside the cocked hat is one in four. This may readily be demonstrated by employing three cords to represent three position lines, each fastened to a board at a position representing one of the observed marks. This would provide a useful piece of equipment in the hands of a teacher in demonstrating errors in three position lines obtained from compass bearings, if his aim is to teach his students rather than merely prepare them for an examination.

The following combinations of errors in laying down position lines from bearings of marks $\mathrm{A}, \mathrm{B}$ and $\mathrm{C}$, apply to Figs. 4 and 5 respectively. $\mathrm{T}$ in both figures represents the true position of the ship. The sense of the error is indicated by (R) (to the right) or (L) (to the left).

Referring to Fig. 4:

$\begin{array}{llllll}\text { Case } 1 & \epsilon_{1}(\mathrm{R}) \epsilon_{2}(\mathrm{R}) \epsilon_{3}(\mathrm{R}) & \text { or } & \epsilon_{1}(\mathrm{~L}) \epsilon_{2}(\mathrm{~L}) \epsilon_{3}(\mathrm{~L}) \\ \text { Case } 2 & \epsilon_{1}(\mathrm{R}) \epsilon_{2}(\mathrm{R}) \epsilon_{3}(\mathrm{~L}) & \text { or } & \epsilon_{1}(\mathrm{~L}) \epsilon_{2}(\mathrm{~L}) \epsilon_{3}(\mathrm{R}) \\ \text { Case } 3 & \epsilon_{1}(\mathrm{R}) \epsilon_{2}(\mathrm{~L}) \epsilon_{3}(\mathrm{~L}) \text { or } & \epsilon_{1}(\mathrm{~L}) \epsilon_{2}(\mathrm{R}) \epsilon_{3}(\mathrm{R}) \\ \text { Case } 4 & \epsilon_{1}(\mathrm{R}) \epsilon_{2}(\mathrm{~L}) \epsilon_{3}(\mathrm{R}) \text { or } & \epsilon_{1}(\mathrm{~L}) \epsilon_{2}(\mathrm{R}) \epsilon_{3}(\mathrm{~L})\end{array}$

Case I T outside cocked hat

Case 2 T outside cocked hat

Case $3 \mathrm{~T}$ outside cocked hat $\mathrm{T}$ inside one case in four

Case $4 \mathrm{~T}$ inside cocked hat

Referring to Fig. 5 :

$\begin{array}{llllll}\text { Case I } & \theta_{1}(\mathrm{R}) \theta_{2}(\mathrm{R}) \theta_{3}(\mathrm{R}) & \text { or } & \theta_{1}(\mathrm{~L}) \theta_{2}(\mathrm{~L}) \theta_{3}(\mathrm{~L}) \\ \text { Case } 2 & \theta_{1}(\mathrm{R}) \theta_{2}(\mathrm{R}) \theta_{3}(\mathrm{~L}) & \text { or } & \theta_{1}(\mathrm{~L}) \theta_{2}(\mathrm{~L}) \theta_{3}(\mathrm{R}) \\ \text { Case } 3 & \theta_{1}(\mathrm{R}) \theta_{2}(\mathrm{~L}) \theta_{3}(\mathrm{~L}) & \text { or } & \theta_{1}(\mathrm{~L}) \theta_{2}(\mathrm{R}) \theta_{3}(\mathrm{R}) \\ \text { Case } 4 & \theta_{1}(\mathrm{R}) \theta_{2}(\mathrm{~L}) \theta_{3}(\mathrm{R}) & \text { or } & \theta_{1}(\mathrm{~L}) \theta_{2}(\mathrm{R}) \theta_{3}(\mathrm{~L})\end{array}$

Case $\mathrm{I}$ T inside cocked hat

Case 2 T outside cocked hat

Case 3 T outside cocked hat $\} T$ inside one case in four

Case 4 T outside cocked hat

It will be clear that whether $\mathrm{T}$ is inside or outside the cocked hat, assuming that one is formed, is dependent wholly upon the combination of the senses of the errors, and in no way depends upon their magnitudes.

It will also be noticed that Fig. 4 illustrates the normal case of fixing when coasting, in which the three marks generally lie more or less on the same straight line. Fig. 5 illustrates the case where the observer lies within the triangle formed by the lines joining the observed marks, and this often applies to vessels at anchor in indented bays. 


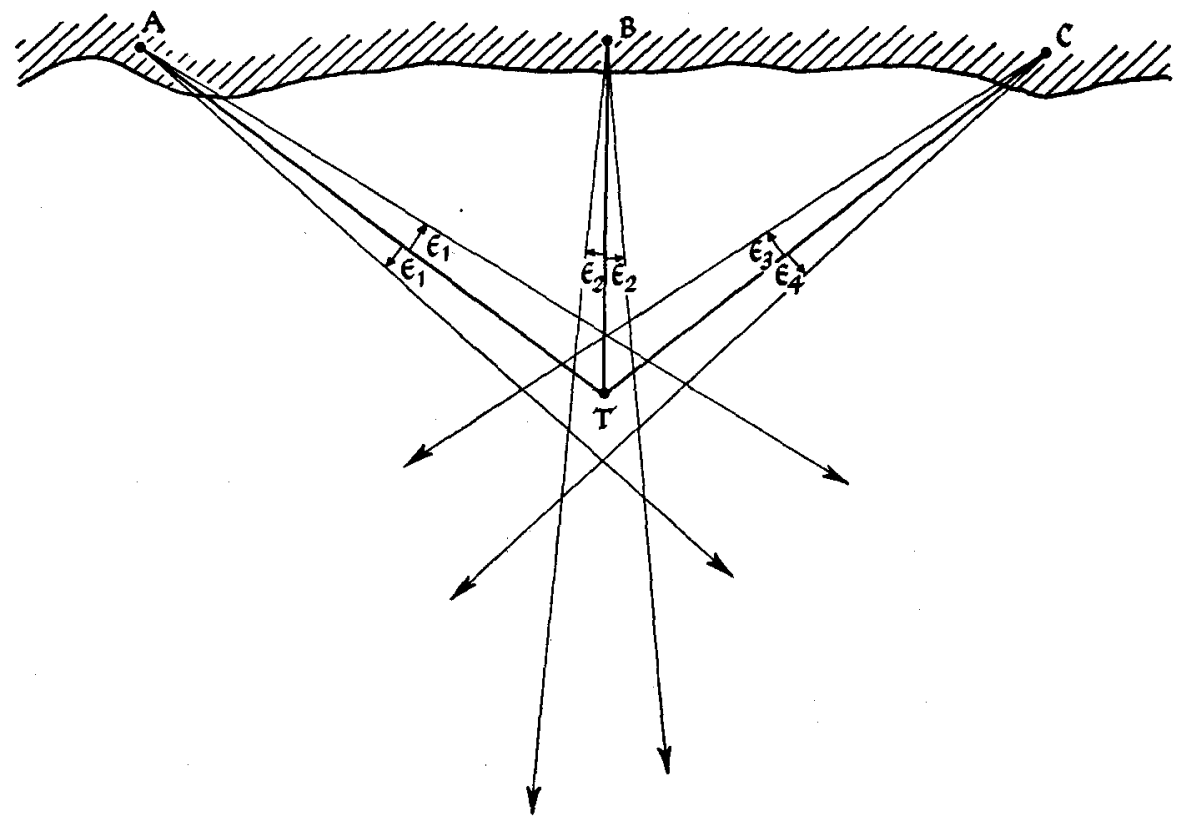

Fig. 4

The conditions pertaining to Fig. 5 provide for a much more reliable fix than those pertaining to Fig. 4 in respect of the angles of cut between the position lines. When marks lie relative to the ship as they do in the conditions illustrated in Fig. 5, the bearings are symmetrical as opposed to the asymmetrical bearings applicable to the conditions illustrated in Fig. 4.

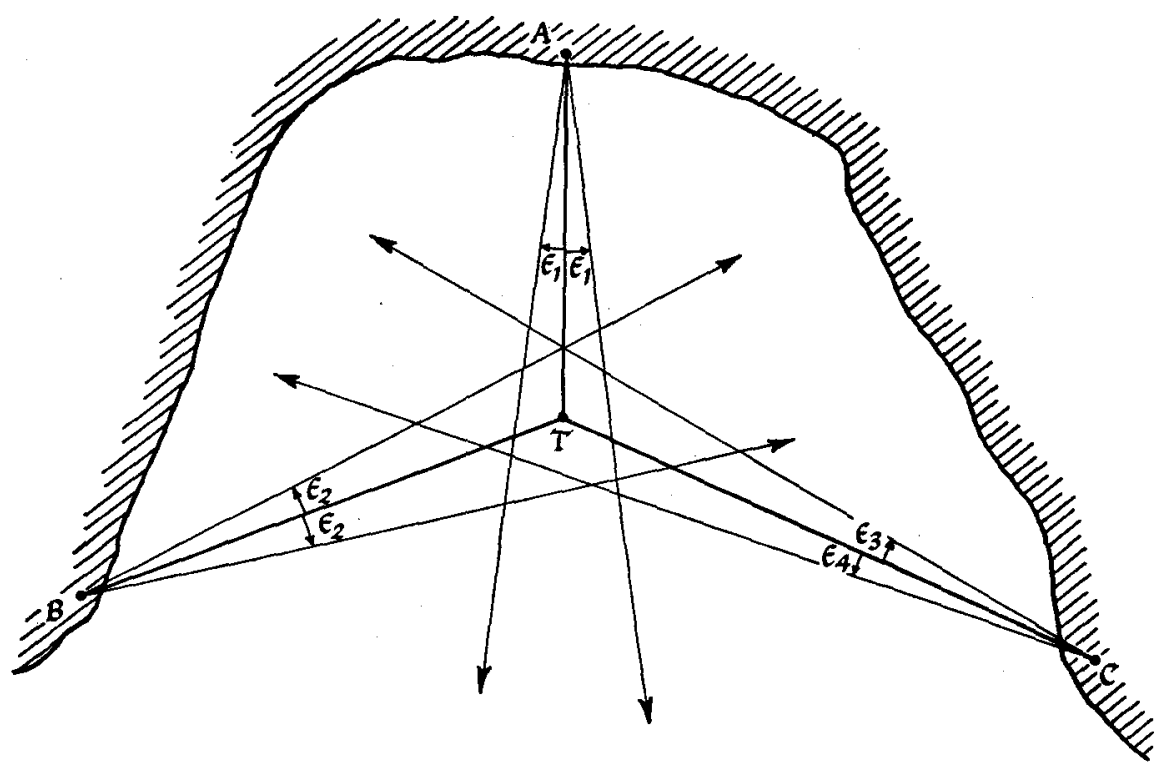

Fig. 5 
It will be noticed from Figs. 4 and 5 that when asymmetrical bearings are observed, the observer's position will be inside the cocked hat if the senses of the errors of the three bearings are not the same but those of the outer marks are different. In the case of three symmetrical position lines the observer's position is within the cocked hat when the errors in the three position lines have the same sense. One conclusion to be drawn from this is that if the errors of the three bearings have the same sense (as would probably be the case if the error were systematic), assuming the ship's position to lie within the cocked hat would be justifiable only if symmetrical bearings were observed. On the assumption that the errors in laying down position lines from compass bearings are probably systematic, and that in the process of conducting a vessel coastwisewhen compass bearings are rarely symmetrical-no justification can be found for assuming a fix to be somewhere inside a cocked hat, if one is formed.

It may readily be seen that in cases where an error in laying down three position lines occurs in one position line only, the observer will lie at one corner of the resulting cocked hat. In cases where an error occurs in two of three position lines, the observer will lie at a point located on one of the sides of the cocked hat.

The chances of an observer lying within a cocked hat, considering that an error may affect one, two, or three position lines which form it, are small indeed-very much less than one in four.

It should be remembered that if a common error affects three position lines, the true position of the observer may be found by using the method of fixing by horizontal angles. Therefore in cases where the compass error is unknown, this method of fixing-using a station pointer if the marks are well-placed-should be employed instead of the method of fixing by cross bearings. It follows that if a cocked hat results in laying down three position lines, the navigator may determine readily whether or not the cocked hat results from a common error in the three position lines. If each of the three pairs of position circles intersect at different corners of the cocked hat, it would be evident that the error in laying down the position lines is common in all three.

We now come to the principal practical problem which faces the navigator when he is confronted with a cocked hat. It is clearly unwise to assume, if the ship is in dangerous waters, that the ship is inside or even on the boundary of the cocked hat. If after applying the position circle test, it is found that the errors in laying down the position lines are random errors, then instead of regarding the results of the three observations as three lines, each should be regarded as a position sector centred at the charted position of the corresponding observed mark, and whose angular width is proportional to the estimated error, plus and minus, in the direction of the ship from the mark. Three such position sectors will in general result in the formation of a six-pointed star, somewhere within which the observer must be located if the actual errors in laying down the position lines are less than the estimated errors.

The spatial extent of this six-pointed star of certainty, is a function of the angles of cut between the position lines. The smaller are these angles the larger will be the star for any given estimated errors in laying down.

Any error in fixing by cross bearings of two marks is proportional to the cosecant of the angle of cut, and when the angle of cut is less than about $30^{\circ}$, any error in laying down the position lines will result in a relatively large error in the fix. If therefore the angles between the bearings of the marks exceed $30^{\circ}-$ as they should - the star of certainty will be relatively small in extent. 
Fixing by horizontal angles is generally to be preferred to fixing by cross bearings, but if this method is not available because available marks are not wellplaced, and a cocked hat results from laying down three position lines obtained from compass bearings, the degree of accuracy of each position line should be estimated and three position sectors drawn. The ship's position should then be estimated to lie at that corner of the star of certainty which will give the safest margin of error.

Many mariners will no doubt argue that drawing stars of certainty on their navigational charts every time a fix is required, is not a practical solution to the problem, and I would tend to agree with them in most cases. However, the above simple treatment serves to emphasize the need for care when setting courses from fixes obtained from three compass bearings. The margin of error allowed should clearly be more than that which would result from assuming the true position of the ship to be at one corner of the cocked hat. Furthermore, the prudent seaman should continue to regard his plotted fixes, if they appear to be perfect, with caution and suspicion.

\title{
Error of the Closest Point of Approach
}

\author{
from N. Sameshima
}

THE problem of errors in radar plotting were considered by Capt. H. Topley (11, 167) and Capt. F. J. Wylie (12, 198), and it was shown that the error in the estimated distance of the C.P.A. depends upon mean range and range change in plotting interval. I want to introduce the term rate of error in the estimated distance of C.P.A., in the same way that Topley shows the percentage error in the estimated speed.

In Fig. $1, O$ is own ship, $A$ is the first plotted point

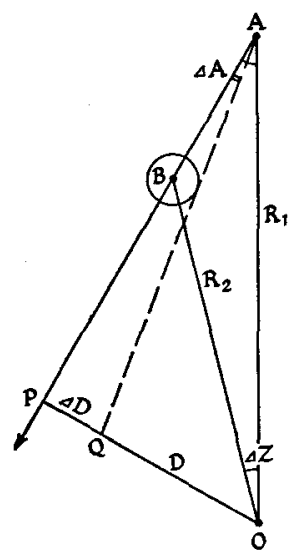

Fig. 1 and $\mathrm{B}$ is the second plotted point. $R_{1}$ and $R_{2}$ are ranges of echoes and $\Delta Z$ is the difference between two bearings. $A B$ is the distance run on the relative track. Hence, $D$ (OP) is the estimated distance of the C.P.A.

Then,

$$
D=\frac{R_{1} R_{2} \sin \Delta Z}{\sqrt{ }\left(R_{1}{ }^{2}+R_{2}{ }^{2}-2 R_{1} R_{2} \cos \Delta Z\right)}
$$

In case of the vessels crossing at a fine-angle, the change of bearing $\Delta Z$ is small, and

$$
D \bumpeq 0.0175 \Delta Z^{\circ} \frac{R_{1} R_{2}}{R_{1}-R_{2}}
$$

From the above formula, the distance of the C.P.A. is proportional to the bearing change and the square of the mean range, and approximately inversely proportional to range change. 